\title{
Evaluation of an Electronic Health Record (EHR) Tool for Integrated Behavioral Health in Primary Care
}

\author{
Katelyn K. Jetelina, PhD, MPH, Tanisha Tate Woodson, PhD, MPH, Rose Gunn, MA, \\ Brianna Muller, BA, Khaya D. Clark, PhD, Jennifer E. DeVoe, MD, DPhil, \\ Bijal A. Balasubramanian, MBBS, PhD, and Deborah J. Cohen, PhD
}

Background: Integrating behavioral health into primary care can improve care quality; however, most electronic health records are not designed to meet the needs of integrated teams. We worked with practices and behavioral health (BH) clinicians to design a suite of electronic health record tools to address these needs ("BH e-Suite"). The purpose of this article is to examine whether implementation of the BH e-Suite changes process of care, intermediate clinical outcomes, and patient experiences, and whether its use is acceptable to practice members and $\mathrm{BH}$ clinicians.

Methods: We conducted a convergent mixed-methods proof-of-concept study, implementing the BH e-Suite across 6 Oregon federally qualified community health centers ("intervention clinics"). We matched intervention clinics to 6 control clinics, based on location and patient panel characteristics, to assess whether process of care (Patient Health Questionnaire-9 [PHQ-9] and Generalized Anxiety Disorder-7 screening) and intermediate outcomes (PHQ-9, Generalized Anxiety Disorder-7 scores) changed postimplementation. Prepost patient surveys were used to assess changes in patient experience. To elucidate factors influencing implementation, we merged quantitative findings with structured observations, surveys, and interviews with practice members.

Results: Implementation improved process of care (PHQ-9 screening). During the course of the study, change in intermediate outcomes was not observed. Degree of BH e-Suite implementation varied: 2 clinics fully implemented, 2 partially implemented, and 2 practices did not implement at all. Initial practice conditions (eg, low resistance to change, higher capacity), process characteristics (eg, thoughtful planning), and individual characteristics (eg, high self-efficacy) were related to degree of implementation.

Conclusions: Health information technology tools designed for behavioral health integration must fit the needs of clinics for the successful uptake and improvement in patient experiences. Research is needed to further assess the effectiveness of this tool in improving patient outcomes and to optimize broader dissemination of this tool among primary care clinics integrating behavioral health. ( $\mathrm{J}$ Am Board Fam Med 2018;31:712-723.)

Keywords: Community Health Centers, Electronic Health Records, Oregon, Patient Health Questionnaire, Primary Health Care, Proof of Concept Study, Surveys and Questionnaires

Each year, 26\% of the United States population experiences an emotional, mental, or behavioral health problem, and the majority of these individ-

This article was externally peer reviewed.

Submitted 5 February 2018; revised 21 May 2018; accepted 25 May 2018.

From Department of Epidemiology, Human Genetics, and Environmental Sciences, UTHealth School of Public Health in Dallas, Dallas, TX (KKJ, BAB); Department of uals are seen in primary care settings. ${ }^{1,2}$ Here we define behavioral health as encompassing "any behavioral problems bearing on health, including mental health and substance abuse conditions,

Family Medicine, Oregon Health \& Science University, Portland, OR (TTW, RG, BM, KDC, JED, DJC); OCHIN, Inc., Portland (JED).

Funding: The National Institute of Mental Health (R34 MH100371) funded this study. 
stress-linked physical symptoms, patient activation, and health behaviors". ${ }^{3}$ Research supports the inseparability of behavioral health care and medical care ${ }^{4-6}$, and strong evidence shows that integration, bringing behavioral and medical care together, improves clinical outcomes, improves patient experiences of care, and reduces health care costs (ie, the "Triple Aim" of health care). ${ }^{7-9}$

Less well known is how to effectively deliver integrated care in routine primary care practice. At many practices beginning to integrate care, a new professional joins the team, a behavioral health clinician (BHC). BHCs are embedded in primary care teams to provide brief, targeted treatment to patients with mild-to-moderate behavioral health conditions. Although most primary care practices now routinely use electronic health records (EHRs), ${ }^{10}$ these EHRs are not tailored to the specific tasks or workflows of BHCs embedded in primary care clinics. Technological improvements are needed to enable BHCs to use the EHR for integrated care delivery. ${ }^{11-13}$

In Turning EHRs into Assets for Mental Health and Uniting Practice (TEAM-UP), we collaborated with OCHIN, a not-for-profit health information network that provides health information technology (IT) solutions to a collaborative of 510 community health centers (CHCs) on a single instance of Epic EHR. In the United States, CHCs comprise the country's health care safety-net by providing primary care and public health services to under or uninsured, low-income patients. A detailed description of OCHIN can be found elsewhere. ${ }^{14,}{ }^{15}$ With input from practicing BHCs, we developed a suite of user-centered tools (the $\mathrm{BH}$ e-Suite) to address the information needs of BHCs practicing in integrated primary care practices. The $\mathrm{BH}$ e-Suite is incorporated into Epic and enables integrated care delivery by organizing the tools BHCs use into a single tab, with fast links to those tools. In addition, we added functionality that supports psychosocial assessment, information-gather-

Conflict of interest: none declared.

Corresponding author: Katelyn K. Jetelina, PhD, MPH, Department of Epidemiology, Human Genetics, and Environmental Sciences, UTHealth School of Public Health in Dallas, Dallas, Texas 6011 Harry Hines Blvd, Suite V8.106C, Dallas, TX (E-mail: katelyn.k.jetelina@uth.tmc.edu).

Prior presentations: Aspects of this analysis were presented at the $44^{\text {th }}$ North American Primary Care Research Group Annual Meeting, Colorado Springs, CO, November 2016. ing, goal-setting, documentation, monitoring, and tracking, by using a range of point-and-click functions and drop-down menus. It also includes templates for commonly used screeners (eg, Patient Health Questionnaire-9 [PHQ-9]; Generalized Anxiety Disorder-7 [GAD-7]) that auto-calculate and auto-populate progress notes and show changes in scores over time.

This study had the following aims:

1. Examine whether implementing the $\mathrm{BH}$ eSuite changes process of care, intermediate clinical outcomes, and patient experiences postimplementation;

2. Assess acceptability of the $\mathrm{BH}$ e-Suite to BHCs; and

3. Identify clinic and practice member factors influencing the implementation of the $\mathrm{BH}$ e-Suite.

We hypothesize that BHCs will find the $\mathrm{BH}$ e-Suite acceptable to use. We also hypothesize that implementing the $\mathrm{BH}$ e-Suite will improve process measures (eg, rate of screening with PHQ-9) and patient experience of care. However, we do not expect improvements in patient depression symptoms (PHQ-9 scores) during the short time frame of this study.

\section{Methods \\ Design}

We used a convergent, iterative, mixed-methods design to accomplish the main aims of this study.

\section{Clinic Sample}

Six OCHIN CHCs (intervention clinics) were recruited to implement and use the $\mathrm{BH}$ e-Suite. A detailed description of the intervention clinics is provided in Table 1. Clinics were offered minimal support to implement the tool (ie, training manual, one 13-minute video) and no financial incentives. Although the functionalities we developed are organized into a single "suite" for BHCs, this functionality and the information documented were available to other users practice-wide. BHCs, however, were the primary users of the $\mathrm{BH}$ e-Suite in this study. Propensity scoring methods were used to match the intervention clinics to control clinics not using the behavioral health tool, based on 7 criteria: (1) geographic location (rural vs urban), (2) race (\% White, log transformed), (3) ethnicity (\% non-Hispanic White, log transformed), (4) insur- 
Clinic 1 (partially implemented) is a primary care Federally Qualified Health Center (FQHC). This practice is partnering with a local nonprofit, community-based prevention, mental health, and addiction agency to colocate one licensed clinical psychologist in the practice 5 days a week. The psychologist provided brief, targeted behavioral health care to patients, with a particular focus on providing behavioral health support to patients with diabetes. Primary care physicians in the practice referred patients to the psychologist, with the front desk scheduling these appointments, and when needed, they engage the psychologist during a patient's visit for an introduction or warm-handoff, or to seek this professional's advice regarding the best treatment path for the patient (eg, see psychologist, see mental health provider, seek specialty treatment outside the practice). The psychologist had a private office in the practice that is located in close proximity to some of the primary care pods and farther from others, as this is a large practice. In addition, this practice also contracted with the same organization to colocate a mental health professional. This professional was an unlicensed social worker and was supervised by a licensed professional located at the mental health organization. This person provided traditional mental health services for patients with more serious and persistent mental illness, typically meeting with patients for 50-minute visits and for 12 weeks or more, as needed. This practice had written protocols in place for introducing the psychologist and mental health provider to patients and for making care transitions/referrals to these professionals. Not long after implementation of the BH e-Suite, the practice lost a critical team member who knew the tool well and was a consistent user. When this individual left, so did the institutional knowledge on tool usage. Although new behavioral health clinicians (BHCs) used the tool, use was superficial (eg, for documentation purposes only).

Clinic 2 (fully implemented) is a primary care FQHC that employs two licensed clinical social workers (LCSW) to provide brief behavioral health care to their patients. The LCSWs shared an office that is adjacent to the primary care team's location in the practice. They did not have a private office to see patients, but generally did so in a medical examination room. Typically, the LCSWs were engaged by a primary care physician when the patient was in for a medical visit; the physician or medical assistant would go find the LCSW, who conducted a brief assessment and intervention and established a plan for following up with the visit. LCSWs would see patients, as needed, for brief visits. These visits are scheduled by the LCSW with the patient. The LCSWs played a central role in screening and brief intervention for substance use, as this had been a practice focus, and had developed protocols and a committee that reviews prescriptions for controlled substances. The practice had clear protocols for these workflows. Prior to expansion of the LCSW workforce in this practice, there was a colocated mental health professional, but this role has been phased out, and LCSWs assisted in transitioning patients to care at a local community mental health center (CMHC) for patients with more serious and persistent mental illness and substance use care need. Tool uptake was initially slow, due in part to internal billing negotiations. Once implemented, additional BHCs joined the staff and all used the tool extensively and fully.

Clinic 3 (did not implement) is a FQHC-CMHC hybrid. It is a county health department health center that includes primary care, mental health care, developmental disabilities services, environmental health, and other health-related community services. The units in this health center functioned autonomously, with the primary care practice located on the 1st floor and the mental health practice located on the 2nd floor. The CMHC served the county and took referrals from other organizations in the county, and primary care physicians in the building referred patients with mental/behavioral needs to the mental health unit in the same way other organizations in the community do. This health center does not currently employ BHCs to work closely with the primary care team. This practice had prior experience embedding BHCs with primary care, but just prior to implementation of the $\mathrm{BH}$ e-Suite these clinicians left the clinic and were not replaced. In addition, this health center does not have written protocols or other documentation in place to describe how medical, behavioral, and mental health care might be integrated for patients. The clinic experienced a great deal of turnover during the study.

Clinic 4 (partially implemented) is a primary care FQHC. This practice partnered with a local nonprofit, community-based prevention, mental health, and addiction agency to colocate one licensed clinical psychologist in the practice 5 days week to provide patients with brief, targeted behavioral health care, with a particular focus on providing behavioral health support to patients with diabetes. The psychologist had a private office and treated patients there, but $s /$ he also had a workstation that is within close proximity to the primary care team. In addition to accepting referrals from the primary care physicians in the practice, physicians engaged the psychologist via warm-handoff so that s/he could provide brief services to patients in the medical examination rooms, as needed. The physicians also sought this professional's advice regarding the best treatment path for patients (eg, in-clinic psychologist, in-clinic mental health provider, out-of-clinic specialty treatment), as needed. The practice also employed a colocated mental health provider who cares for patients with more serious and persistent mental illness who need longer visits (50-minutes) and a longer treatment course. This role was filled by a psychology practicum student from a local university where s/he received supervision for this work. This practice developed workflow for their integrated approach, specifying the roles for the front desk, medical assistants, physicians, psychologists, and mental health provider. At the time the $\mathrm{BH}$ e-Suite was launched, the practice experienced turnover among clinical staff that was quite disruptive. Operations did stabilize and the BH e-Suite was used by BHCs, but these professionals did not use all functionality.

Clinic 5 (fully implemented) is a primary care FQHC that employed one unlicensed social worker (MSW) to provide brief behavioral health care to their patients. The MSW shared an office with the practice's Epic specialist. This professional did not see patients in their office but in medical examination rooms. Typically, the MSW was engaged by a primary care physician when the patient was in for a medical visit; the physician or medical assistant will go find the MSW, who will do a brief assessment and intervention and establish a plan for following up. MSW also helped when patients need to be referred to outpatient mental health and substance use care. MSW would see patients, as needed, for brief visits. These visits were scheduled by the MSW with the patient. In addition, the MSW played a central role in screening and brief intervention for substance use, as this has been a practice focus. The practice had clear protocols for these workflows. The BHC role was new to this practice. Tool implementation was limited in part due to access issues (the BHC had difficulty in accessing certain functionality). Documentation was the primary focus of tool usage. 
ance (\% Medicaid), (5) percent of adults with serious mental health disease (log transformed), (6) number of active primary care providers, and (7) number of active patients. Thus, 6 control clinics were similar to intervention clinics on the above factors but did not use the $\mathrm{BH}$ e-Suite.

\section{Patient Sample}

Eligible patients were frequent attenders (defined as those who were in 75 th percentile for the number of outpatient visits), over 18 years old, diagnosed with at least one behavioral and one medical condition, and seen for a behavioral health visit 12 months before and after implementation of the $\mathrm{BH}$ e-Suite.

\section{Measures and Data Collection}

Process of care measures included the proportion of patients screened for PHQ-9 and GAD-7. Intermediate clinical outcome measures included PHQ-9 and GAD-7 scores. These measures were extracted for eligible patients from the 6 intervention and 6 control clinic EHRs 12 months before and after implementation.

Patient experience-of-care measures included previously validated patient-reported measures of care coordination (Picker Institute Scale, scores range 1 to 3 and 1 to 5$),{ }^{16}$ satisfaction with care (scores range 1 to 5), integration of care (Primary Care Assessment Tool, scores range 1 to 3$),{ }^{17}$ and care communication (Mercer Connecting, Assessing, Responding, and Empowering [CARE] Tool, scores range 1 to 5 ; see Appendix). ${ }^{18}$ These measures were collected by surveying a sample of eligible patients among the intervention clinics at baseline and again at 6 months postimplementation. Surveys were administered in the waiting room by either a research assistant or by a front desk staff person and placed in a secure lockbox after collection to ensure anonymity. An alert within the EHR informed clinic administration that the patient was eligible for a survey. At baseline, 337 patients responded to surveys and postimplementation, 302 patients responded to surveys.

Measures of $\mathrm{BH}$ e-Suite acceptability included domains from the Technology Acceptance Model (TAM), ${ }^{19}$ a 16 -item survey completed by BHCs at baseline and postimplementation that measured the following: (1) Perceived usefulness, including the degree to which the EHR aligned with BHCs' current workflow and tasks and also improved job performance, ability to manage patient care (eg, assess and diagnose patients), organize patient information, and coordinate care with integrated care team members; (2) perceived ease of use, including the perceived burden, time-efficiency, learnability, and interface qualities (ability to navigate and enter information); and (3) percent usability, including the frequency at which the BHCs found themselves abandoning the $\mathrm{BH}$ e-Suite, and the extent to which they used all the available $\mathrm{BH}$ e-Suite features.

\section{Factors Influencing Implementation}

Practice capacity for change was measured using the validated 23-item Adaptive Reserve questionnaire, ${ }^{20-22}$ which was collected among intervention clinics via a practice member survey at baseline. Practice member surveys were distributed at monthly all-staff clinic meetings at baseline and had a greater than $75 \%$ response rate across practices. Qualitative methods (observations and interviews) were used to assess factors influencing implementation of the $\mathrm{BH}$ e-Suite. Observations were conducted by a field team experienced in qualitative research, primary care delivery, informatics, and human factors research. This team conducted a 2-to-4-day site visit at the 6 intervention clinics over a 6-month period, November 2013-April 2014, before implementation of $\mathrm{BH}$ e-Suite. The length of visit depended on practice size, and each visit involved intensive observation of the inte- 
grated care team's EHR use, including observing individual work areas, team work areas, and encounters with patients. In addition, we shadowed primary care clinicians, BHCs (ie, licensed clinical psychologists and social workers), and other key members of the clinical care team, and sat in on their visits with patients when permitted. Among all 6 participating clinics, we accumulated a total of 184 single-spaced pages of field notes from approximately 150 person-hours of observing all aspects of clinical care.

Semistructured interviews were conducted at clinic site visits with 2 to 4 practice members representing different roles in the practice (eg, BHC, primary care providers, medical assistants) to understand their approach to integrating care, the workflows and tasks involved in integration, and how they used the EHR for documentation and information sharing. By observing and interviewing different roles in the practices, we gained a robust understanding of how care for behavioral health conditions was delivered at baseline and the environment in which the $\mathrm{BH}$ e-Suite would be implemented. We also conducted 6-month postimplementation interviews with $\mathrm{BHCs}$ to ascertain the level of implementation, experience with the tool, and changes in clinical practice connected to tool use. Interviews followed a semistructured guide ${ }^{23}$ that assessed integrated care workflows and tasks, experience with the current EHR, practice structure, and organizational culture. We conducted 29 interviews (11 BHCs, 4 medical assistants, 7 mental health clinicians [MHCs], and 7 primary care clinicians), each lasting between 45 minutes and 1 hour. In intervention practices, MHCs provide care for patients with more severe and persistent mental health issues, while BHCs provide care to patients with mild to moderate mental health problems over a shorter period of time.

\section{Data Analysis}

Descriptive statistics (tabulations, percentages, means, and standard deviations) were used to describe EHR-derived process of care and intermediate clinical outcome measures, stratified by case and control clinics at baseline. We used SAS PROC MIXED ${ }^{24}$ to model 2-level, mixed effects (random intercept) regression to account for the clustered sampling design (patients clustered within clinics). Linear regression models were designed to evaluate the interactive relationship between pre- and postimplementation and intervention/control in regard to patient PHQ-9 and GAD-7 scores (dependent variable) across intervention and control clinics. Practice member and patient surveys were descriptively analyzed by examining response frequency distributions. Among intervention clinics, $t$ tests were computed to examine the mean change after implementation in patient-reported outcome measures (eg, care coordination, care communication with primary care physician, integration of care, and satisfaction with care). When specific survey items were not answered, respondents' items were excluded from analyses. Missing data ranged from $1 \%$ to $12 \%$ among patientreported constructs.

Our multidisciplinary team used a 4-step approach to analyze qualitative data. ${ }^{25}$ First, we collectively read field notes and interview data collected from each practice to develop an understanding of each practice, as a case, with particular attention to factors influencing implementation of the tool, BHC workflows, tasks, and tool ease-of-use. Through this process, we created a codebook to label text. We used these codes in group analyses until we reached stability; code definitions were standardized and analysts were trained to code data reliably. Individuals completed the data analysis, meeting as a group to compare work and come to consensus when analytic differences emerged.

Second, we analyzed findings across clinics to identify common themes. We grouped emerging findings into categories of themes by using an immersion-crystallization approach. ${ }^{26}$ Third, we connected our findings to the existing literature. ${ }^{25} \mathrm{We}$ conducted a second in-depth comparative analysis by using the Consolidated Framework for Implementation Research (CFIR) ${ }^{27}$ constructs to understand differences in implementation across intervention clinics. After considering all 39 CFIR constructs, 7 relevant CFIR constructs that mapped to 3 domains (inner setting, process characteristics and individual characteristics) were identified and an in-depth analysis was conducted to identify how these factors influenced implementation of the $\mathrm{BH}$ e-Suite.

Qualitative and quantitative analyses were first conducted independently to ensure unbiased interpretation. After completing analyses, qualitative and quantitative findings were integrated at the practice level by using data-triangulation techniques. ${ }^{25}$ The Institutional Review Board at Ore- 
Table 2. Patient Sample Description of Intervention and Control Clinics at Baseline

\begin{tabular}{|c|c|c|}
\hline & $\begin{array}{c}\text { Intervention, } \\
\mathrm{N}=4,377\end{array}$ & $\begin{array}{c}\text { Control, } \\
\mathrm{N}=3,628\end{array}$ \\
\hline Description & $\mathrm{N}(\%)$ & $\mathrm{N}(\%)$ \\
\hline Age, mean, SD & $49.1(14.2)$ & $49.1(14.6)$ \\
\hline Sex, female & $1,308(30)$ & $1,113(30)$ \\
\hline \multicolumn{3}{|l|}{ Race/Ethnicity } \\
\hline White, Non-Hispanic & $2,964(69)$ & $2,302(65)$ \\
\hline Black, Non-Hispanic & $36(1)$ & $101(3)$ \\
\hline Hispanic & $1,216(28)$ & $990(28)$ \\
\hline Other & $86(2)$ & $170(5)$ \\
\hline Language, English & $3,280(75)$ & $2,792(77)$ \\
\hline \multicolumn{3}{|l|}{ Household income, \$ } \\
\hline$<14,999$ & $2,254(56)$ & $2,219(63)$ \\
\hline 15,000 to 29,999 & $1,339(33)$ & $835(24)$ \\
\hline 30,000 to 44,999 & $319(8)$ & $277(8)$ \\
\hline 45,000 to 59,999 & $77(2)$ & $113(3)$ \\
\hline $60,000+$ & $36(1)$ & $71(2)$ \\
\hline \multicolumn{3}{|l|}{ Number of comorbidities } \\
\hline 0 & $455(10)$ & $356(10)$ \\
\hline 1 & $1,008(23)$ & $791(22)$ \\
\hline 2 & $1,107(25)$ & $910(25$ \\
\hline $3+$ & $1,807(41)$ & $1,571(43)$ \\
\hline Depression diagnosis & $1,951(45)$ & $1,678(46)$ \\
\hline Diabetes diagnosis & $1,175(27)$ & $1,122(31)$ \\
\hline
\end{tabular}

SD, standard deviation.

gon Health \& Science University approved this study protocol (No. 9366).

\section{Results}

The distribution of patient characteristics was similar between intervention and control clinics (Table 2).

Intervention clinics implemented the $\mathrm{BH}$ e-Suite to varying degrees: two practices used all the features and functions of the BH e-Suite EHR tool ("fully implemented"); 2 practices implemented some, but not all, of the features of the tool ("partially implemented"); and 2 practices did not use the tool at all ("did not implement").

\section{Clinical Care and Patient Perception of Care}

Overall, there was a significant increase between intervention and control clinics in process of care, specifically the proportion of patients screened with PHQ-9 (Table 3). There was no change in intermediate clinical outcome measures (ie, PHQ-9 and GAD-7 scores). When examined by degree of implementation, significant but small differences were observed in outcome measures between intervention and control clinics. Table 3 also displays the change in patient-reported experience of care postimplementation. Patient perceptions of integration-of-care improved overall and among clinics that fully implemented the tool. Patient perceptions of communication with primary care providers significantly improved only among clinics that partially implemented the tool.

\section{Acceptability of BH e-Suite}

Clinicians working in clinics that fully or partially implemented the $\mathrm{BH}$ e-Suite reported that the tool was acceptable and easy to use. They reported the tool added 1 to 2 minutes to the initial visit but saved a significant amount of time during follow-up visits by automatically populating the history of the presenting illness and patient instructions at subsequent visits. As a BHC from clinic 5 who fully implemented the tool reported:

"[Tool adoption was] actually really easy because we were a small, very small core group of people when we started, and as we brought on new hires the system adopted it. Our IT people adopted it, our Epic people adopted it and it is not like you have a choice. This is just what we're doing. We're using this. As we've hired new folks, this has just been what they have been introduced to and it is been great."

BHC reported finding the tool beneficial, too:

"I would say the biggest gain for me has been in follow-up visits so I am not having to copy paste from previous visits. It is really streamlined the process for follow-up in terms of mental status examination and history of the presenting illness. Those parts have helped immensely" (Clinic 2).

Clinics that fully or partially implemented the tool found it easy to use (mean Technology Acceptance Model score, 3.75 and 3.5, respectively). Among clinics that partially implemented the $\mathrm{BH}$ e-Suite, clinicians reported that they would use the tool more often if they were given more training in its use. For instance, a newly hired BHC at Clinic 1 who only used the tool for documenting encounters, explained that she was just learning to use Epic and picking up the $\mathrm{BH}$ e-Suite as well as learning to practice in a new setting was difficult: "I just stopped using it [all] because... it just seemed like a lot... it just takes time and practice, but some days my schedule is just really full, and I just have to go quickly." All clinics that implemented the tool to 
Table 3. Clinical Care and Patient Experience of Care Outcomes across Degree of Implementation

\begin{tabular}{|c|c|c|c|c|}
\hline & Overall & Fully Implemented & Partially Implemented & Did Not Implement \\
\hline \multicolumn{5}{|l|}{ Process of Care Outcomes* } \\
\hline PHQ-9 screens, OR & $1.06^{\ddagger}$ & $1.10^{\ddagger}$ & 1.01 & $1.11^{\ddagger}$ \\
\hline GAD-7 screens, OR & 1.01 & 1.01 & $0.85^{\S}$ & $1.03^{\S}$ \\
\hline \multicolumn{5}{|l|}{ Intermediate Clinical Outcomes* } \\
\hline PhQ-9 scores, $\beta$ & 0.16 & -0.64 & 0.23 & $1.98^{\ddagger}$ \\
\hline GAD-7 scores, $\beta$ & 1.38 & 2.59 & 4.42 & $-4.55 \|$ \\
\hline \multicolumn{5}{|c|}{ Patient Experiences of $\mathrm{Care}^{\dagger}$, mean (SD) } \\
\hline Satisfaction with care & $0.06(0.07)$ & $0.09(0.11)$ & $0.08(0.12)$ & $-0.03(0.08)$ \\
\hline Care communication with PCP & $0.15(0.08)$ & $-0.02(0.13)$ & $0.39^{\S}(0.13)$ & $0.04(0.13)$ \\
\hline Care communication with $\mathrm{BHC}$ & $0.06(0.13)$ & $0.10(0.24)$ & $0.12(0.23)$ & $-0.10(0.20)$ \\
\hline Integration of care & $0.20^{\S}(0.07)$ & $0.44^{\ddagger}(0.13)$ & $0.09(0.13)$ & $0.10(0.13)$ \\
\hline Care coordination & $0.06(0.04)$ & $0.06(0.06)$ & $0.11(0.06)$ & $-0.02(0.07)$ \\
\hline
\end{tabular}

BHC, behavioral health clinician; GAD-7, Generalized Anxiety Disorder-7; PCP, primary care provider; PHQ-9, Patient Health Questionnaire-9; OR, odds ratio; SD, standard deviation.

*Pre/post change among intervention clinics compared to control clinics.

${ }^{\dagger}$ Pre/post change among intervention clinics.

${ }^{\ddagger} P$ value $<.001$.

${ }^{\$} P$ value $<.01$.

$\| P$ value $<.05$.

some degree reported an increase in perceived usability from preintervention (mean, 1.87) to postintervention (mean, 3.78) (data not shown).

\section{Factors Influencing Implementation of the BH e-Suite}

Clinics that fully implemented the BH e-Suite had higher adaptive reserve scores, indicating greater capacity for change (Table 4). They also had leadership buy-in and support, more resources dedicated to implementation, processes and workflows developed in advance of implementation, staff of all levels engaged in implementation, and BHCs who were formally trained in use of the $\mathrm{BH}$ e-Suite. BHCs noted that the tool simplified EHR use, and they welcomed it as a way to help address their health IT needs. The lead BHCs championed tool implementation and supported staff use of the tool by creating step-by-step guides, allocating time and space for training and fostering collaboration among BHCs and Epic specialists. Clinics that partially implemented had less adaptive capacity and needed more time, training, and support to use the tool. They also experienced important disruptive events (turnover in BHC staff), which was a barrier to implementation. Clinics that did not implement the $\mathrm{BH}$ e-Suite had the lowest adaptive capacity and also lacked leadership buy-in and support for use of the BH e-Suite. Importantly, although these clinics agreed to participate in the study, saying they would have an embedded BHC on staff, they were unable to fulfill this promise and, instead, continued to refer patients to a colocated MHC who had different information and documentation needs; MHCs at these clinics used a different EHR tool to meet their unique documentation needs.

\section{Discussion}

Policy reforms have been implemented across the United States in support of the integration of behavioral health services into primary care clinics, ${ }^{28}$ and there are an increasing number of initiatives aimed at integrating care across diverse settings. ${ }^{29-31}$ This momentum to change practice and policy is crucial because integrated care is specifically designed to identify and address patient medical and behavioral health care needs together and to improve quality, patient experience, and reduce costs. ${ }^{4,6,32-35}$

Systems that have integrated, or are planning to integrate, behavioral health and medical care must consider an investment in health IT. Documentation requirements for BHCs are different from other members on the integrated team. Therefore, clinics must think about workflows, tasks, and health IT functionality to effectively use the EHR to deliver high quality integrated primary care. Our 
Table 4. Implementation Degree across Practices and Consolidated Framework for Implementation Research Domains

\begin{tabular}{|c|c|c|c|c|}
\hline Domain & Definition & Fully Implemented & Partially Implemented & Did Not Implement \\
\hline \multicolumn{5}{|l|}{ Inner Setting } \\
\hline $\begin{array}{l}\text { Baseline Adaptive } \\
\text { Reserve (mean, SD) }\end{array}$ & $\begin{array}{l}\text { Practice capacity } \\
\text { for change }\end{array}$ & $0.63(0.08)$ & $0.56(0.07)$ & $0.55(0.08)$ \\
\hline \multirow[t]{3}{*}{$\begin{array}{l}\text { Implementation } \\
\text { climate }\end{array}$} & $\begin{array}{l}\text { Commitment to } \\
\text { implement } \\
\text { change }\end{array}$ & $\begin{array}{l}\text { - } \text { BHCs interested in } \\
\text { improving BH } \\
\text { documentation in EHR }\end{array}$ & $\begin{array}{l}\text { - } \mathrm{BHC} \text { interested in } \\
\text { improving BH } \\
\text { documentation in } \\
\text { EHR }\end{array}$ & $\begin{array}{l}\text { - BHCs satisfied with current } \\
\text { system and resistant to new } \\
\text { tool }\end{array}$ \\
\hline & & $\begin{array}{l}\text { - Upper management } \\
\text { advocated for using a } \\
\text { new tool tailored to } \\
\text { BHC needs }\end{array}$ & $\begin{array}{l}\text { - Interest in tool from } \\
\text { upper management }\end{array}$ & $\begin{array}{l}\text { - Little buy-in from upper } \\
\text { management }\end{array}$ \\
\hline & & $\begin{array}{l}\text { - Epic specialist available } \\
\text { to assist with } \\
\text { implementation. }\end{array}$ & $\begin{array}{l}\text { - Epic site specialist did } \\
\text { not assist with } \\
\text { implementation }\end{array}$ & $\begin{array}{l}\text { - Integration model did not } \\
\text { facilitate use of the tool, as } \\
\text { these clinics focused on } \\
\text { long mental health } \\
\text { appointments, rather than } \\
\text { brief interventions with } \\
\text { BHCs }\end{array}$ \\
\hline $\begin{array}{l}\text { Readiness for } \\
\text { implementation }\end{array}$ & $\begin{array}{l}\text { Organizational } \\
\text { capacity to } \\
\text { implement } \\
\text { change }\end{array}$ & $\begin{array}{l}\text { - Step-by-step guides } \\
\text { produced by BHC } \\
\text { leadership on how to } \\
\text { use the tool } \\
\text { - Stable BHC workforce, } \\
\text { written BHC } \\
\text { workflows in place }\end{array}$ & $\begin{array}{l}\text { - Unstable BHC } \\
\text { workforce/turnover; } \\
\text { no written BHC } \\
\text { workflows }\end{array}$ & $\begin{array}{l}\text { - No time allocated for } \\
\text { training and educating } \\
\text { BHCs about tool although } \\
\text { Epic site specialist available }\end{array}$ \\
\hline \multicolumn{5}{|l|}{$\begin{array}{l}\text { Process } \\
\quad \text { Character }\end{array}$} \\
\hline \multirow[t]{2}{*}{ Planning } & $\begin{array}{l}\text { Tasks and methods } \\
\text { conducted to } \\
\text { prepare for } \\
\text { implementation }\end{array}$ & $\begin{array}{l}\text { - Had introductory } \\
\text { implementation } \\
\text { meeting before rolling } \\
\text { out the tool }\end{array}$ & $\begin{array}{l}\text { - No implementation } \\
\text { plan aside from one } \\
\text { introductory } \\
\text { implementation } \\
\text { meeting before rolling } \\
\text { out the tool }\end{array}$ & $\begin{array}{l}\text { - Had introductory } \\
\text { implementation meeting } \\
\text { before rolling out tool }\end{array}$ \\
\hline & & $\begin{array}{l}\text { - Lead BHC acted as } \\
\text { opinion leader and } \\
\text { champion of tool } \\
\text { implementation }\end{array}$ & $\begin{array}{l}\text { - No follow-up by } \\
\text { clinics to implement }\end{array}$ & $\begin{array}{l}\text { MH manager felt the tool } \\
\text { was not useful to them and } \\
\text { declined implementing the } \\
\text { tool }\end{array}$ \\
\hline Engaging & $\begin{array}{l}\text { Involvement of staff } \\
\text { in the change } \\
\text { process }\end{array}$ & $\begin{array}{l}\text { Familiarized other } \\
\text { clinical staff, including } \\
\text { front desk staff and } \\
\text { PCPs, with the new } \\
\text { tool }\end{array}$ & $\begin{array}{l}\text { - BHC supervisor left } \\
\text { this role; loss of } \\
\text { champion derailed } \\
\text { implementation }\end{array}$ & $\begin{array}{l}\text { - No clinic champion or } \\
\text { formally appointed internal } \\
\text { implementation leader }\end{array}$ \\
\hline $\begin{array}{l}\text { Reflecting and } \\
\text { evaluating }\end{array}$ & $\begin{array}{l}\text { Team debriefing } \\
\text { about process and } \\
\text { experience }\end{array}$ & $\begin{array}{l}\text { Dedicated agenda time } \\
\text { at BHC monthly } \\
\text { meeting to discuss the } \\
\text { tool and provide } \\
\text { feedback to tool } \\
\text { developer }\end{array}$ & $\begin{array}{l}\text { - Feedback given to } \\
\text { tool developers, but } \\
\text { no follow through by } \\
\text { practice }\end{array}$ & $\begin{array}{l}\text { - No reflection or evaluation } \\
\text { because tool was not } \\
\text { implemented }\end{array}$ \\
\hline \multicolumn{5}{|l|}{$\begin{array}{l}\text { Individual } \\
\text { Characteristics }\end{array}$} \\
\hline \multirow[t]{2}{*}{$\begin{array}{l}\text { Knowledge/beliefs } \\
\text { about intervention }\end{array}$} & $\begin{array}{l}\text { Individuals' } \\
\text { attitudes toward } \\
\text { and value placed } \\
\text { on intervention }\end{array}$ & $\begin{array}{l}\text { - Believed tool enhanced } \\
\text { EHR use (simplified it) }\end{array}$ & $\begin{array}{l}\text { - Newly hired BHCs } \\
\text { reported receiving } \\
\text { limited (1 hour), and } \\
\text { inadequate training in } \\
\text { the tool }\end{array}$ & $\begin{array}{l}\text { - Did not find the tool to be } \\
\text { useful }\end{array}$ \\
\hline & & $\begin{array}{l}\text { - Consensus view: tool } \\
\text { was a step of progress }\end{array}$ & $\begin{array}{l}\text { - BHCs did not know } \\
\text { how to use tool }\end{array}$ & \\
\hline Self-efficacy & $\begin{array}{l}\text { Belief in one's } \\
\text { ability to execute } \\
\text { implementation } \\
\text { tasks }\end{array}$ & $\begin{array}{l}\text { - } \mathrm{BHCs} \text { felt they } \\
\text { received sufficient } \\
\text { training to use tool }\end{array}$ & & \\
\hline
\end{tabular}

$\mathrm{BH}$, behavioral health; $\mathrm{BHC}$, behavioral health clinician; $\mathrm{MH}$, mental health; $\mathrm{PCP}$, primary care provider; EHR, electronical health record; SD, standard deviation. 
study showed that a user-centered suite of EHR tools tailored for BHCs was feasible to use and acceptable to embedded BHCs practicing in integrated CHCs. Importantly, use of the $\mathrm{BH}$ e-Suite increased the perceived productivity of BHCs and improved process of care. At the outset of this study, we were sensitive to the unintended effects of having BHCs use the EHR during patient visits, as this might negatively impact the therapeutic relationship, patient experience of care, and intermediate clinical outcomes. With regard to patient experiences of care, we found the opposite to be true; use of the $\mathrm{BH}$ e-Suite improved patient perceptions of communication with their clinicians and BHCs, as well as their perceptions of receiving integrated care. Through in-depth observation, interviews, and the intentional engagement of users in the design process, we successfully developed a tool that was acceptable to patients and BHCs and avoided a common error of overcomplicating documentation for short BHC visits. We believe that this careful design work may have contributed to the $\mathrm{BH}$ e-Suite's ability to create care efficiencies and patient connection, without compromising the therapeutic relationship.

Our study has important lessons for clinics planning to use health IT tools for integration. In order for clinics to invest the time to implement the $\mathrm{BH}$ e-Suite, these tools must fit their needs. Clinics that had a "colocated" approach to care (patients internally referred to specialty MHCs for longer-term care instead of to an integrated $\mathrm{BHC}$ ) did not find the $\mathrm{BH}$ e-Suite fitting. In fact, these clinics had a competing set of tools, specifically designed by and for specialty MHCs; not surprisingly, these clinics preferred the other tools. This suggests that motivation for implementation and perceived usefulness of a tool are aligned and must be carefully considered during health IT implementation.

Two study clinics with high motivation and adaptive capacity implemented these tools on their own and subsequently demonstrated improvement in patient experience. Two clinics that had midlevel adaptive capacity were only able to partially implement the $\mathrm{BH}$ e-Suite, despite recognizing its utility. These latter 2 clinics likely represent the norm among primary care clinics in the United States, which suggests the need for evidence-based implementation support strategies, such as practice facilitation or expert consultation, to assist with tool implementation. This finding has implications for clinics and researchers considering a health IT implementation; regardless of the improvements using a new health IT tool may bring, successful implementation may require external assistance.

\section{Limitations}

Our results should be considered in light of several limitations. First, the sample size of clinics was small, and generalizability of the study findings is limited. However, even with the small number of clinics, there was considerable variability in the integration and implementation approach, and we found that use of the $\mathrm{BH}$ e-Suite significantly enhanced patient experiences of care. Second, although we recognized the social and technical aspects of the change process when introducing a new tool within primary care, ${ }^{36}$ this study did not have the resources to provide implementation support to clinics, and we did not test how to best implement the $\mathrm{BH}$ e-Suite. Our qualitative findings provide formative insights into the factors that influence implementation, but more work is needed to study the effectiveness as well as the wider dissemination and implementation of this tool among CHCs. Third, some may consider it a limitation that we developed this tool in Epic, as this EHR system may not be affordable for all clinics. However, the partnership between researchers, OCHIN Epic developers, and CHCs made development of this tool possible. OCHIN serves over $500 \mathrm{CHCs}$ across the nation, and they all now have access to the $\mathrm{BH}$ e-Suite. We have published details about the functionality of these tool elsewhere, allowing for this suite of tools and features to be developed by others ${ }^{23}$ and streamlining behavioral health integration.

Integrated primary care clinics need EHR tools that support the delivery of whole-person care. EHRs are seldom designed with behavioral health in mind; to date, few EHR-based tools have been developed and tested to support the work of BHCs and integrated teams. The $\mathrm{BH}$ e-Suite may fill this need, as it was acceptable to BHCs, feasible to implement by motivated clinics with some training, and has the promise to improve outcomes. More research is needed to assess the effectiveness of this tool in improving process and/or patient outcomes that are not just limited to depression symptoms but also to manage chronic diseases (such as type 2 diabetes) where integrated care has been shown to have an impact. Furthermore, future research 
should investigate how to disseminate and implement this tool widely among integrated primary care clinics.

We would like to express our deep gratitude with the clinics that participated in this pilot study. Without their participation, this study would have been impossible. In addition, we would like to recognize the input of Benjamin Miller, $\mathrm{PhD}$, Timothy Burdick, $\mathrm{MD}, \mathrm{MS}$, and Rodger Kessler, $\mathrm{PhD}$, all whom shared their expertise throughout the course of this study. We would also like to thank Amanda Delzer Hill for providing editorial assistance.

To see this article online, please go to: bttp://jabfm.org/content/ 31/5/712.full.

\section{References}

1. Kessler RC, Demler O, Frank RG, et al. Prevalence and treatment of mental disorders, 1990 to 2003. N Engl J Med 2005;352:2515-23.

2. Regier DA, Narrow WE, Rae DS, Manderscheid RW, Locke BZ, Goodwin FK. The de facto U.S. Mental and Addictive Disorders Service System: epidemiologic catchment area prospective 1-year prevalence rates of disorders and services. Arch Gen Psychiatry 1993;50:85-94.

3. Peek CJ and National Integration Academy Council. Lexicon for behavioral health and primary care integration: concepts and definitions developed by expert consensus. Rockville, MD: Agency for Healthcare Research and Quality; 2013.

4. Butler M, Kane RL, McAlpin D, et al. Integration of mental health/substance abuse and primary care: evidence report/technology assessment no. 173. Rockville, MD: Agency for Healthcare Research and Quality; 2008.

5. Craven M, Bland R. Better practices in collaborative mental health care: an analysis of the evidence base. Can J Psychiatry 2006;51(6 Suppl 1):7S-72S.

6. deGruy F. Mental health care in the primary care setting. In: Donaldson MS, Yordy KD, Lohr KN, Vanselow NA, editor. Primary care: America's health in a new era. Washington, DC: Institute of Medicine; 1996.

7. Andrews G. Should depression be managed as a chronic disease? BMJ 2001;322:419-21.

8. Katon W, Sullivan MD. Depression and chronic medical illness. J Clin Psychiatry 1990;51(Suppl):3-11.

9. Berwick DM, Nolan TW, Whittington J. The triple aim: care, health, and cost. Health Aff 2008;27: 759-69.

10. Cifuentes M, Davis M, Fernald D, Gunn R, Dickinson P, Cohen DJ. Electronic health record challenges, workarounds, and solutions observed in practices integrating behavioral health and primary care. J Am Board Fam Med 2015;28(Suppl 1):S63-S72.

11. Collins C, Hewson DL, Munger R, Wade T. Evolving Models of Behavioral Health Integration in Pri- mary Care. New York, NY: Milbank Memorial Fund; 2010.

12. Shank N, Willborn E, PytlikSillig L, Harmoni Joie N. Electronic health records: eliciting behavioral health providers' beliefs. Community Mental Health J; 2011;48:249-54.

13. US Department of Health and Human Services. Mental health research findings. Rockville, MD: Agency for Healthcare Research and Quality; 2009.

14. DeVoe JE, Sears A. The OCHIN community information network: bringing together community health centers, information technology, and data to support a patient-centered medical village. J Am Board Fam Med 2013;26:271-8.

15. DeVoe JE, Gold R, Spofford M, et al. Developing a network of community health centers with a common electronic health record: description of the Safety Net West Practice-based Research Network (SNW-PBRN). J Am Board Fam Med 2011;24:597604.

16. Sizmur S, Redding D. Core domains for measuring inpatient's experience of care. Oxford, UK: Picker Institute; 2009.

17. Shi L, Starfield B, Xu J. Validating the Adult Primary Care Assessment Tool. J Fam Practice 2001; 50:161-75.

18. Mercer SW, Maxwell M, Heaney D, Watt GC. The consultation and relational empathy (CARE) measure: development and preliminary validation and reliability of an empathy-based consultation process measure. Fam Pract 2004;21:699-705.

19. Davis FD, Bagozzi RP, Warshaw PR. User acceptance of computer technology: a comparison of two theoretical models. Manage Sci 1989;35:9821003 .

20. Nutting PA, Crabtree BF, Stewart EE, et al. Effect of facilitation on practice outcomes in the National Demonstration Project model of the patient-centered medical home. Ann Fam Med 2010;8(Suppl 1):S33-S44.

21. Miller W, Crabtree B, Nutting P, Stange K, CR J. Primary care practice development: a relationshipcentered approach. Ann Fam Med 2010;8(Suppl 1): S68-S79.

22. Jaen CR, Crabtree BF, Palmer RF, et al. Methods for evaluating practice change toward a patient-centered medical home. Ann Fam Med 2010;8(Suppl 1):S9-20; s92.

23. Woodson TT, Gunn R, Clark KD, et al. Designing health information technology tools for behavioral health clinicians integrated within a primary care team. Journal of Innovation in Health Informatics. In press.

24. SAS Institute Inc. SAS 9.4. Cary NC. SAS Institute Inc.; 2014.

25. Crabtree BF, Miller WL. Chapter 4: Key informant interviews. In: Doing qualitative research. Thousand Oaks, CA: SAGE Publications; 1999. P. 82. 
26. Borkan J. Immersion/crystallisation. In: Crabtree $\mathrm{BF}$, Miller W. Doing qualitative research. Thousand Oaks, CA: Sage; 1999. P. 179-194.

27. Damschroder LJ, Aron DC, Keith RE, Kirsh SR, Alexander JA, Lowery JC. Fostering implementation of health services research findings into practice: a consolidated framework for advancing implementation science. Implement Sci 2009;4:1-15.

28. Goodson JD. Patient Protection and Affordable Care Act: promise and peril for primary care. Ann Intern Med 2010;152:742-4.

29. Bachrach D, Anthony S, Detty A. State strategies for integrating physical and behavioral health services in changing Medicaid environment. Washington, D.C.: The Common Wealth Fund; 2014.

30. Cohen DJ, Davis MM, Gunn R, et al. Integrating behavioral health and primary care: consulting, coordinating and collaborating among professionals. J Am Board Fam Med 2015;28(Supp 1):S21-S31.

31. Cohen DJ, Balasubramanian BA, Davis $M$, et al. Understanding care integration from the ground up: five organizing constructs that shape integrated prac- tices. J Am Board Fam Med 2015;28(Suppl 1):S7S20.

32. Balasubramanian BA, Cohen DJ, Jetelina KK, et al. Outcomes of integrated behavioral health with primary care. J Am Board Fam Med 2017;30:130-9.

33. Davis MM, Balasubramanian BA, Cifuentes M, et al. Clinician staffing, scheduling, and engagement strategies among primary care practices delivering integrated care. J Am Board Fam Med 2015;28(Suppl 1):S32-40.

34. Gilbody S, Bower P, Fletcher J, Richards D, Sutton AJ. Collaborative care for depression: a cumulative meta-analysis and review of longer-term outcomes. Arch Intern Med 2006;166:2314-21.

35. Petterson SM, Phillips RL, Jr., Bazemore AW, Dodoo MS, Zhang X, Green LA. Why there must be room for mental health in the medical home. Am Fam Physician 2008;77:757.

36. Ludwick DA, Doucette J. Adopting electronic medical records in primary care: lessons learned from health information systems implementation experience in seven countries. Int J Med Inform 2009;78: 22-31. 


\begin{tabular}{|c|c|}
\hline Measure & Survey Questions \\
\hline \multirow[t]{8}{*}{$\begin{array}{l}\text { Care coordination using the } \\
\text { Picker Institute Scale }\end{array}$} & $\begin{array}{l}\text { 1) Did you know who was in charge of your care for each of } \\
\text { your health problems? }\end{array}$ \\
\hline & $\begin{array}{l}\text { 2) How often were the doctors, nurses and other health care } \\
\text { providers who cared for you familiar with your most } \\
\text { recent medical history? }\end{array}$ \\
\hline & $\begin{array}{l}\text { 3) How often were your providers aware of changes in your } \\
\text { treatment that other providers recommended? }\end{array}$ \\
\hline & $\begin{array}{l}\text { 4) Do you think your providers had all the information they } \\
\text { needed, such as test results, to make decisions about your } \\
\text { treatment? }\end{array}$ \\
\hline & $\begin{array}{l}\text { 5) How often did you know who to ask when you had } \\
\text { questions about your health problems? }\end{array}$ \\
\hline & $\begin{array}{l}\text { 6) How often were you given confusing or differing } \\
\text { information about your health or treatments? }\end{array}$ \\
\hline & $\begin{array}{l}\text { 7) How often did you know what the next step in your care } \\
\text { would be? }\end{array}$ \\
\hline & 8) How well did your health care providers work together? \\
\hline
\end{tabular}

Satisfaction with care

Integration of care using the Primary Care Assessment Tool

Care communication using the Connecting, Assessing, Responding, and Empowering (CARE Tool) you think you need it;
How satisfied have you been with ...

1) the overall quality of care at your primary care clinic;

2) being able to get an appointment in your clinic as soon as

3) being able to get a telephone call or email answered from your primary care clinic as soon as you think you need it.

1) Did your primary care doctor suggest you go to the behavioral health provider?

2) Did your doctor discuss with you different places you could have gone to get help with that problem?

3) Did your doctor or someone working with your doctor help you make the appointment for that visit?

4) Did your doctor provide any information for the behavioral health provider about the reason for the visit?

5) Did the doctor know you made this visit to the behavioral health provider?

6) Did your doctor know what the results of this visit were?

7) After going to the behavioral health provider, did your doctor talk with you about what happened at the visit?

8) Does your doctor seemed interested in the care you get from the behavioral health provider?

How was the doctor at...

[1] =Poor, $[2]=$ Fair, $[3]=$ Good, [4]= Very good, $[5]=$ Excellent

$[1]=$ Very dissatisfied, [2] $=$ Dissatisfied, [3]= Neutral, [4]= Satisfied, $[5]=$ Very satisfied

\footnotetext{
[1] $=$ Probably Not, $[2]=$ Probably, and [3]= Definitely
}
1) making you feel at ease
2) letting you tell your story
3) really listening
4) being interested in you as a whole person
5) fully understanding your concerns
6) showing care and compassion
7) being positive
8) explaining things clearly
9) helping you take control
10) making a plan of action with you

Questions 1 to $4:[1]=$ No, $[2]=$ Yes, sometimes, 3]= Yes, most of the time; or

Questions 5 to 8: [1]=Never, $[2]=$ Rarely,

$[3]=$ Occasionally, $[4]=$

Often, [5]= Very often 